\title{
Applications of the Dollfus Polarization Modulator in Stellar Polarimetry
}

\section{J. TINBERGEN (Leiden)}

"An astronomer is someone to whom a factor of 10 is important, but a factor of 2 is not." If we agree about this, we may say that stellar polarization is $1 \%$ and that we wish to measure that polarization to $1 \%$ accuracy. Polarimeters therefore need an accuracy of $0,01 \%$ (of the total intensity).

In general, stellar polarimetry has been rather a delicate measurement. This is because its nature is differential, i. e. the information required is the small difference between two large observables. There are two general classes of polarimeter, single-detector and twodetector. In the single-detector polarimeters the observables are measured one after the other by the same detector and time-varying attenuations cause errors. In two-detector polarimeters, differences between the two channels cause errors. In both cases extreme stability is required. The solution is to use the single-detector type, but to alternate between the two observables faster than any of the time-varying attenuations. This type of polarimeter uses some sort of polarization modulation.

Though modulation polarimeters differ in the details of their action, the principle they have in common is that they convert the polarization information into an intensity modulation of a beam of constant state of polarization. Traditionally, stellar polarimetry is done with only very few filters, because all such filters have to be calibrated for their polarization properties. However, once the beam is intensity-modulated, any kind of wavelength discriminator may be used without introducing errors. Since we often want to use multi-channel instruments for efficiency, there is a demand for white-light polarization modulators.

The Dollfus modulator (DOLLFUS 1963, MARIN 1965) is in my view the best modulator in existence at present; it is shown in Fig. 1. It is a relatively narrow-band instrument. 1 have been working on the development of a white-light version of this modulator (a description of this work will be submitted to "Astronomy and Astrophysics"). My calculations convince me that the range 3000 to $10000 \AA$ can be covered with a single modulator, to yield a precision of $0,01 \%$ of the total intensity (given sufficient light; I am concerned only with systematic instrumental errors). I can provide details on request.

In order to test my calculations, I designed a prototype using plastic waveplates in achromatic combinations. Optically the instrument is of indifferent quality, yet $I$ reach the precision quoted above; the range is only 4000 to $7000 \AA$ (optically; the detector cuts off at $6000 \AA$ ). Fig. 2 and 3 show some of the results, which agree with those of BEHR, HILTNER and other experienced observers. The polarimeter itself is stable and convenient to use, and the electronics do not require any special care during construction or use. The results have given me confidence in my calculations.

The reason I am taking up your time with a technical talk is that I wish to stress that it is technically feasible to design modulators which can convert any photometer or spectrometer into a polarimeter with the precision quoted, at the expense of half the light and a trebling (roughly) of the electronics. I would like to ask observers and astrophysicists to think hard whether polarization observations might not solve some of their problems for them. Technically there is no real problem; it is a matter of manpower and money. Dr. SERKOWSKI has shown us how useful spectropolarimetry can be, in variable star research particularly.

\section{References :}

DOLLFUS, A., 1963, Comptes Rendus Acad. Sci. Paris 256, 1920.

MARIN, M., 1965, Revue d'Optique 44, 115. 


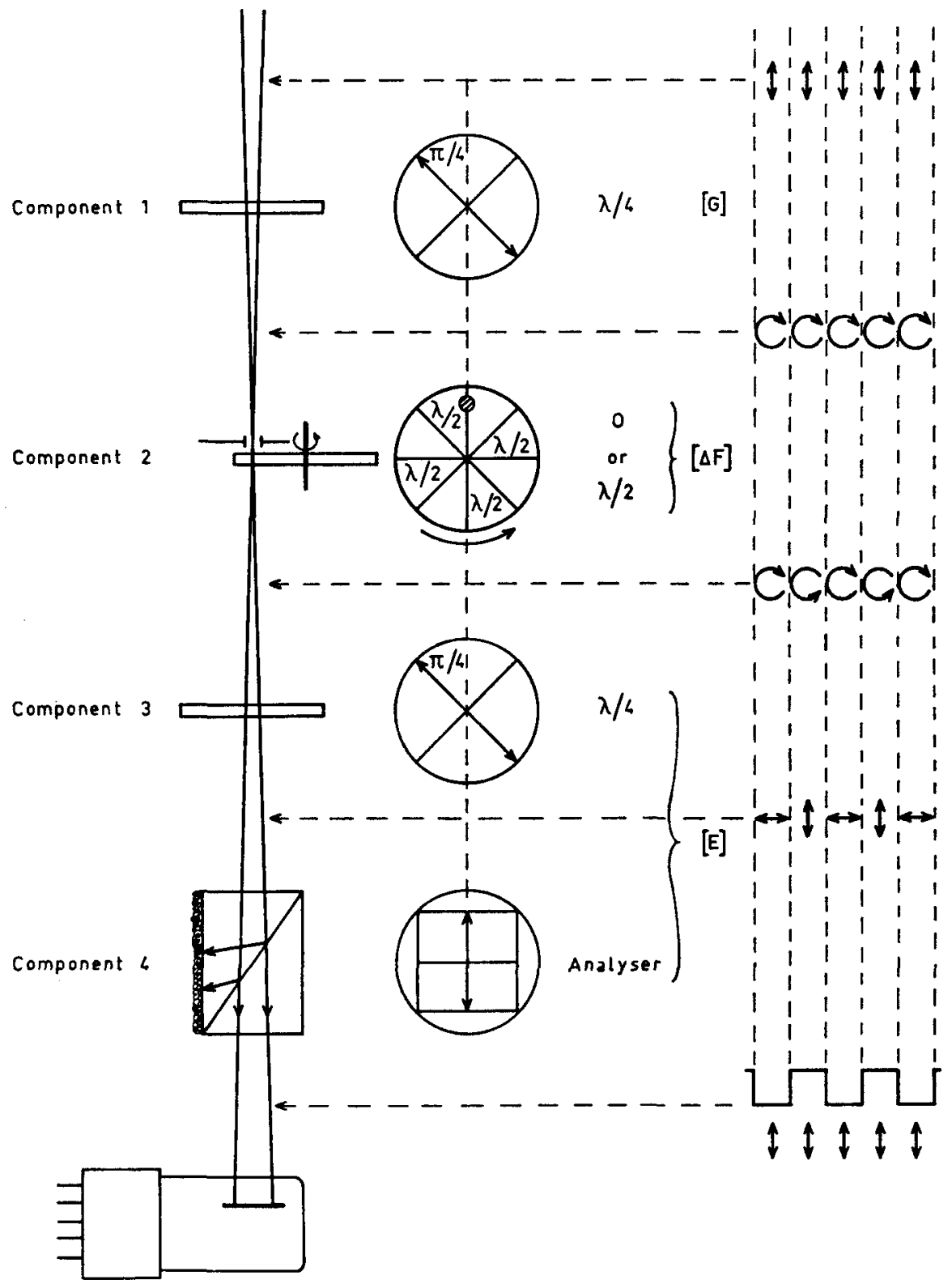

Fig. 1: Schematic representation of the Dollfus polarimeter. The fast axis of each component (transmission axis in the case of the analyser) is indicated by the double-ended arrows. 


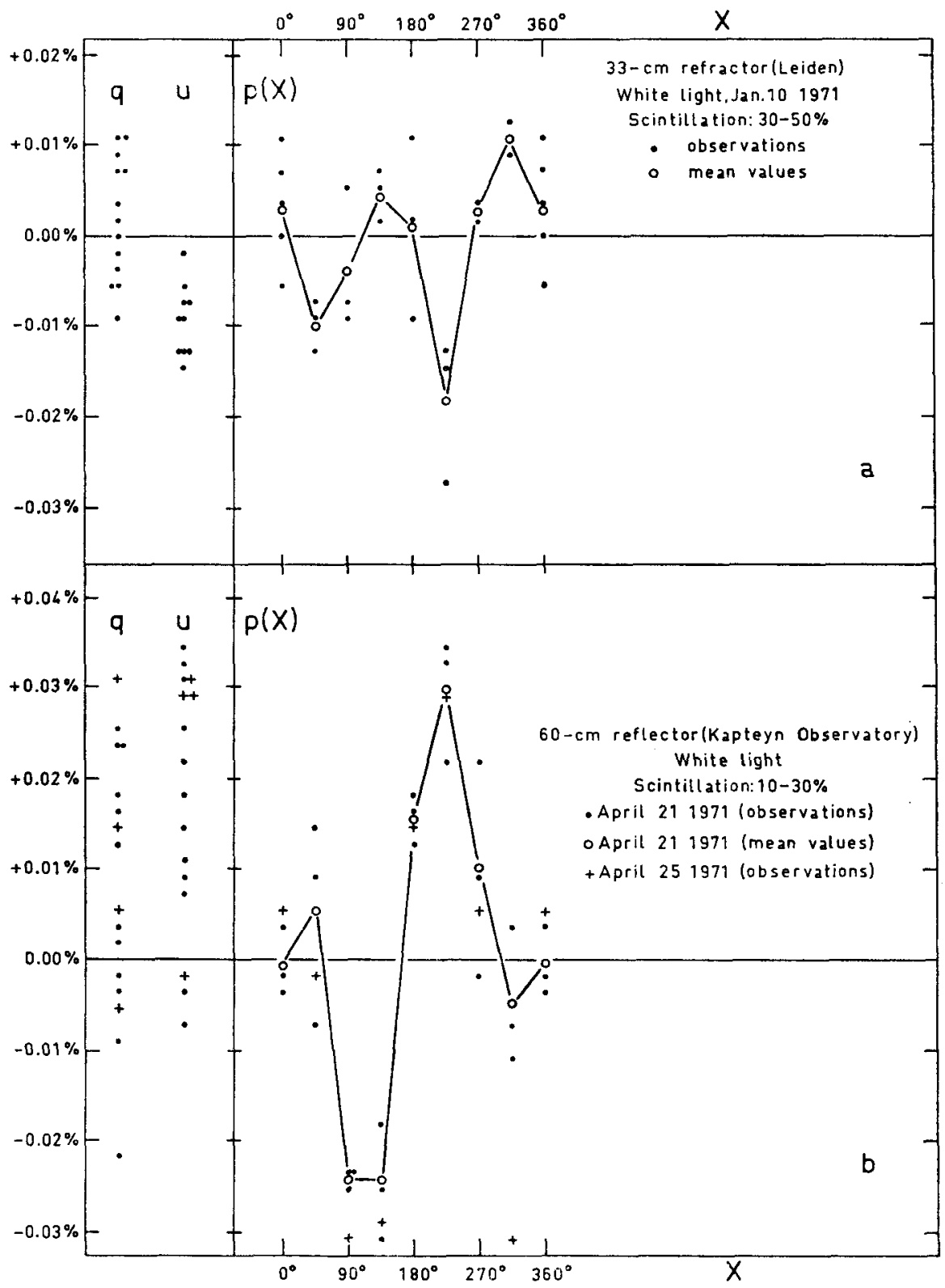

Fig. 2: Observations of a star (Arcturus) with near-zero polarization, represented by $p(X)$ and Stokes parameters $q$ and $u ; q=p\left(0^{\circ}\right)=-p\left(90^{\circ}\right)=p\left(180^{\circ}\right)=-p\left(270^{\circ}\right)$; $\mathrm{u}=\mathrm{p}\left(45^{\circ}\right)=-\mathrm{p}\left(135^{\circ}\right)=\mathrm{p}\left(225^{\circ}\right)=-\mathrm{p}\left(315^{\circ}\right) . \mathrm{X}$ is the position angle of component 1 . 

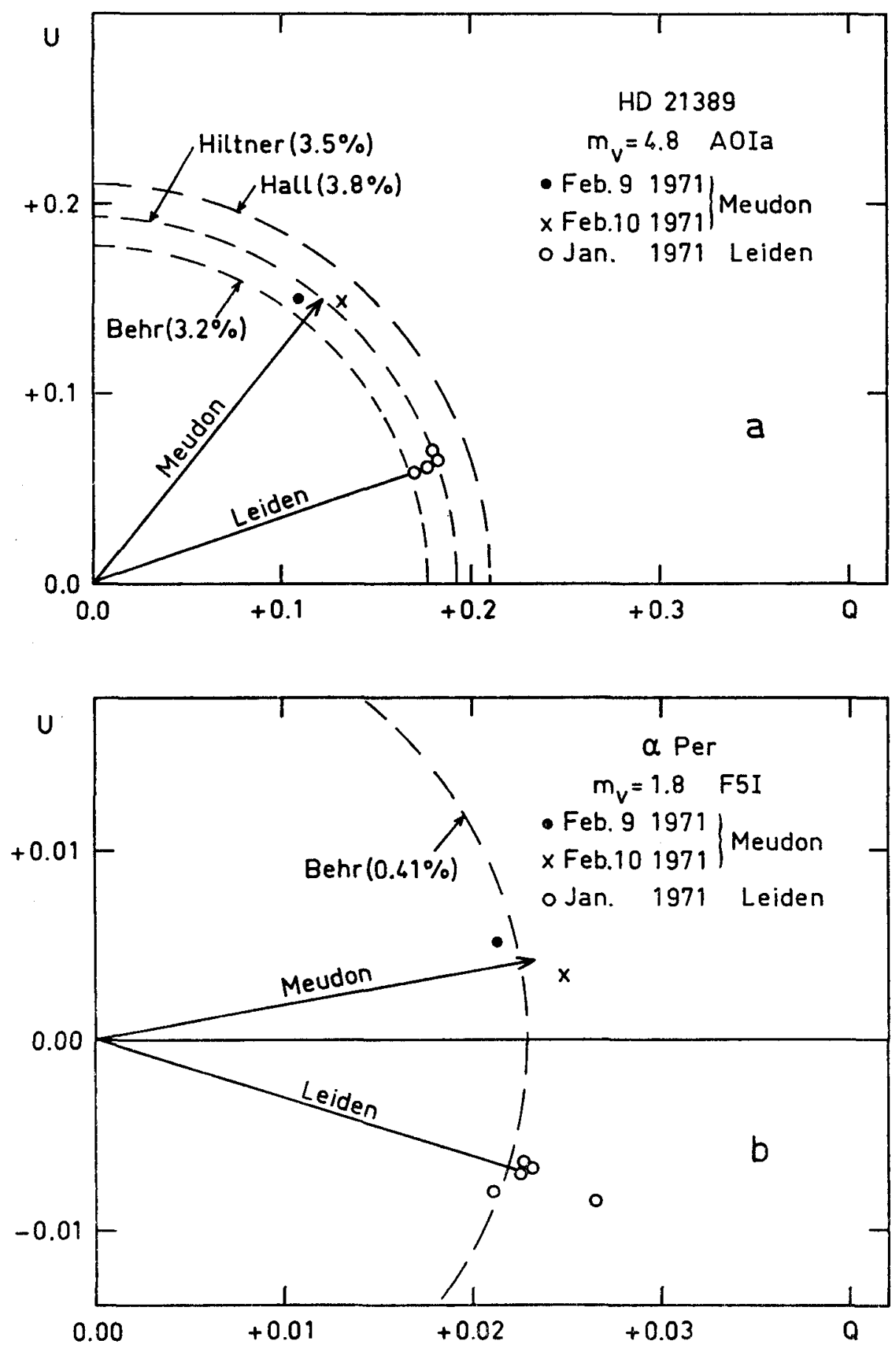

Fig. 3: Observations in white light of two polarized stars with the $33-\mathrm{cm}$ refractor at Leiden and the $60-\mathrm{cm}$ reflector at Meudon. Each point represents the mean for one night. The instrumental systems have astronomically arbitrary orientations in both cases; instrumental polarization in Meudon was about $0,03 \%$ and was corrected for in the observations of $\alpha$ Per. The circles represent the degree of polarization as listed by various observers. 


\title{
Discussion to the paper of TINBERGEN
}

SERKOWSKI: At how many positions of the first quarter-wave plate should the observations be made and how the linear polarization produced by reflection from the birefringent material of quarter-wave plate is eleminated?

TINBERGEN: In theory 3 positions are sufficient to determine 2 Stokes parameters plus an instrumental zero. In practice I use 4, but this is an observational detail, not really important from the instrumental point of view. Analysis of the DOLLFUS polarimeter shows that dichroism of the front element is not important, physically the reason is that it produces only linear polarization at a point in the equipment where the information is contained in the circular polarization.

BEHR: With all our 2 cell polarimeters at Göttingen and at ESO in Chile the limiting factor was finally the changing sensitivity of the photocathodes in the order of $10^{-4}$. To search this limit or even reach higher accuracy, the proposed way looks quite promising.

\section{Polarization of Young Shell Variables}

\section{BREGER (Stony Brook)}

\begin{abstract}
Stellar polarization between 3000 and $7500 \AA$ has been measured in NGC 2264. Out of 37 stars (including many variables) 4 are definitely polarized, while 3 others show weak polarization. The mean (interstellar) cluster polarization is found to be very small. The highest polarization $(4,6 \%)$ is found in a $T$ Tauri star. W 90 , a variable below the main sequence, has the highest polarization found among the $A$ and $F$ stars $(2,6 \%)$. With the exception of W 46, polarization peaks in the blue implying small particle sizes. A good correlation is found between the size of polarization and (other) shell characteristics. These measurements confirm the recently proposed shells in some pre-main sequence stars which appear to be too faint for their color, but fail to detect any new stars with extensive shells. This suggests that some of the scatter in the color-magnitude diagram amongst the $A$ and F stars is real because of an age spread between 1 and $3 \times 10^{6}$ years.

This is the first of two reports on our investigation of the incidence, variability and composition of circumstellar shells amongst pre-main sequence stars in NGC 2264.

Shell stars often appear polarized due to dust particles aligned in a weak magnetic field or scattering in asymmetric gas or dust distributions. If we expect some correlation between the size of the shell and the polarization of light passing through it, then polarization measurements of the stars in NGC 2264 should help determine the incidence of shells. Such polarization measurements can only be interpreted once a correction for the interstellar contribution is made (if large enough). NGC 2264 is ideally suited for these measurements since the interstellar polarization can be determined from the unpolarized stars in the cluster. Furthermore, the amount of interstellar reddening between us and the cluster has been shown to be small, so that the interstellar polarization should also be small. Finally, membership of the cluster stars has been studied.

The Kitt Peak National Observatory polarimeter attadhed to the No. 1 36-inch telescope at the Kitt Peak National Observatory was used during eleven nights in January and February, 1971. The polarimeter utilizes a star-sky chopper and a rapidly rotating polarizer. The data is stored in 400 channels. Instrumental polarization was determined from standard stars with near-zero polarization. It was less than $2,0 \%$ in all colors and was subtracted out. Four different filters were used. The UBV filter system used as well as the other equipment, operation and reduction have recently been described by DYCK and SANFORD (1971). Our 7000 A filter consists of a $1 \mathrm{~mm}$ RG-5 filter with a shortward cutoff near $6500 \mathrm{~A}$.
\end{abstract}

